\title{
Thermal Conductivity of Graphite Felt at High Temperatures
}

\author{
Khaled Chahine ${ }^{*}$, Mark Ballico ${ }^{*}$, John Reizes ${ }^{* *}$ and Jafar Madadnia ${ }^{* *}$ \\ *National Measurement Institute \\ P O Box 264, Lindfield, NSW 2070 \\ ${ }^{* *}$ University of Technology, Sydney \\ P O Box 123, Broadway, NSW 2007 \\ Email : $\underline{\text { Khaled.Chahine@measurement.gov.au }}$
}

\begin{abstract}
Thermal conductivity measurements in vacuum, helium and air of WDF graphite felt were conducted at room temperature. It was found that conduction along the solid paths, gas conduction and radiation between fibres are the dominant heat transfer mechanisms. All heat transfer models reviewed indicated that there are geometrical parameters to be determined experimentally in order to be able to quantify the conduction and radiative mechanisms. Experimental results obtained at room temperature were used to calculate the conduction tortuosity, $\tau$. Results from the literature were used to determine the radiation constant, $C_{f r}$. Using these parameters, an equation for the felt thermal conductivity as a function of the absolute temperature was obtained.
\end{abstract}

\section{Introduction}

At the National Measurement Institute, graphite felts purged with helium, argon or nitrogen are used as insulators for the $48 \mathrm{~kW}$ Thermogage high temperature blackbody furnace described in $[1,2]$. To improve this standard, numerical modelling was used. Values for the thermal conductivity of this graphite felt at different temperatures were determined for use in the numerical models.

Preliminary investigations of the felt datasheet supplied by the manufacturer showed the thermal conductivity to be inconsistent. Experiments were conducted to measure the thermal conductivity of graphite felt in vacuum, air and helium at room temperature. These results are used to calculate different geometrical factors of the felt, which are important in the determination of the felt thermal conductivity.

\section{Experimental Work}

The felt used in our application is a graphite felt grade WDF manufactured by National Specialty Product USA [3], and its density is given as $80 \mathrm{~kg} / \mathrm{m}^{3}$. The radius of the graphite fibres was measured at the National Measurement Institute using a projector magnifier. The measurements were carried out on a sample of fibres extracted from the felt randomly. The results showed that the radius, $r$, of the fibres varies between 7.0 and $12.5 \mu \mathrm{m}$. The resultant average radius was $10.5 \mu \mathrm{m}$ with a $3.2 \mu \mathrm{m}$ standard deviation.

The bulk density of graphite was obtained from the literature with values between 1500 and $1770 \mathrm{~kg} / \mathrm{m}^{3}$ [4]. The volume fraction, $f$, of the felt is defined as the ratio of the felt density to the bulk material density, and therefore
$4.5 \%<f<5.5 \%$. For the remainder of this paper, the value of $f$ is taken to be $5.0 \%$.

The thermal conductivity of the WDF felt measured in vacuum and argon, as given in the manufacturer's datasheet, is plotted in Figure 1. It is expected that the difference between the vacuum and the argon thermal conductivities given by the two graphs in Figure 1 should amount to the argon thermal conductivity, or very close to it. However, examination of these graphs showed that this difference is too high by a factor of between 2 and 12, rendering these data unsuitable for use.

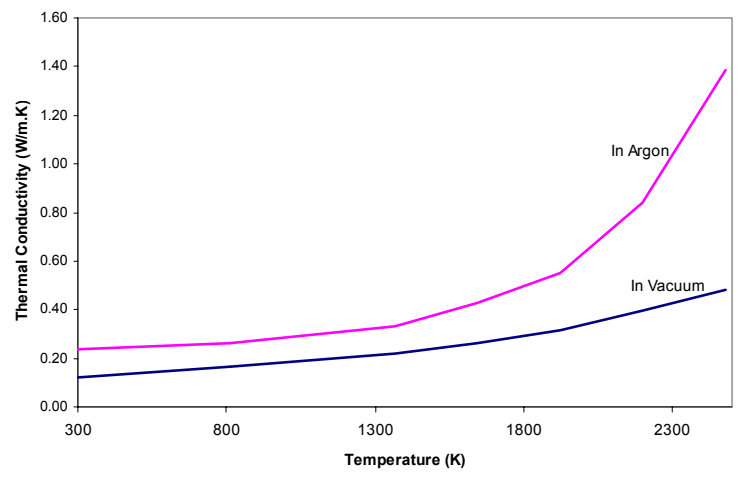

Fig. 1. Thermal conductivity of WDF felt versus temperature in argon and vacuum given by the National Specialty Products USA.

Measurements of the thermal conductivity of WDF felt were conducted at our laboratory using the "Hot-Wire" technique [5]. This technique was used because of its suitability for materials with low thermal conductivity. A copper wire was sandwiched between two layers of WDF felt, each layer being $80 \mathrm{~mm}$ long, $50 \mathrm{~mm}$ wide and $10 \mathrm{~mm}$ thick. A current of $7.00 \mathrm{~A}$ was passed through the wire causing its temperature to rise. The rise in temperature, $\Delta T$, was obtained by measuring the voltage increase in the copper wire at two points $55 \mathrm{~mm}$ apart, and using values for the copper temperature coefficient of resistance to convert the voltage readings to temperatures.

The thermal conductivity, $k$, was calculated from the solution to the transient heat conduction equation [5]

$$
\Delta T=\frac{P}{4 \pi k}\left(\ln \left(\frac{4 \alpha t}{d^{2}}\right)-C\right)
$$

where $P$ is the power per unit length, $\alpha$ is the thermal diffusivity, $t$ is the time, $d$ is the distance between the contact points where the voltage is measured, and $C$ is a constant. 
Figure 2 is a plot of the temperature rise versus time. The readings in the first few seconds were affected by the initial transients establishing the temperature field in and around the copper wire, and therefore they were discarded. The straight line section of the plot indicated that heat was transferred in the felt only. The slowing of the temperature rise was taken to indicate that heat transfer had reached the edge of the felt. Therefore, only the linear section of the graph was used to calculate $k$.

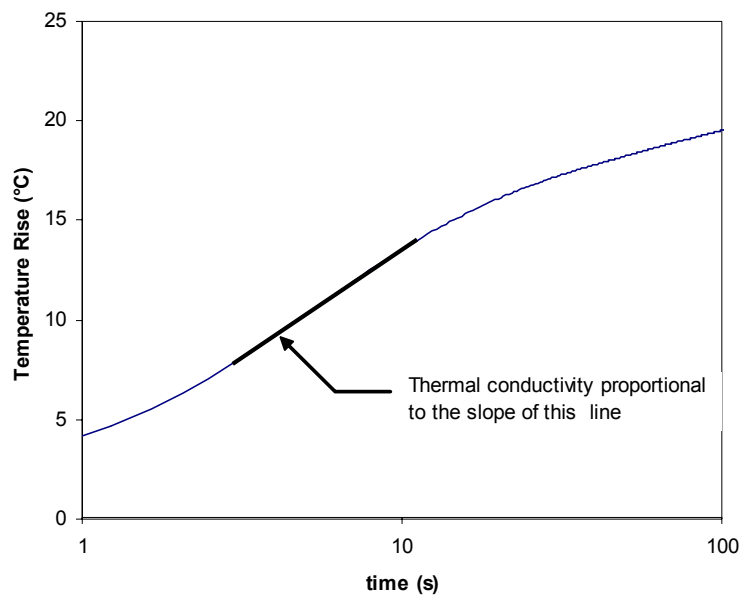

Fig. 2. Rise in temperature due to heating of a copper wire sandwiched inside WDF graphite felt.

Measurements were carried out in vacuum, helium and air. Air was used because it is readily available and has similar thermal conductivity to nitrogen and argon. Thermal conductivity of WDF felt was measured only at room temperature due to our limited capacity to carry out measurements at higher temperatures. The results obtained are presented in Table 1.

\begin{tabular}{|c|c|c|}
\hline Measured in & $\begin{array}{l}\text { Thermal Conductivity } \\
\mathbf{W m}^{-1} \mathrm{~K}^{-1}\end{array}$ & $\begin{array}{c}\text { Standard Deviation } \\
\mathbf{W m}^{-1} \mathbf{K}^{-1}\end{array}$ \\
\hline Vacuum & 0.064 & 0.003 \\
\hline Air & 0.077 & 0.003 \\
\hline Helium & 0.232 & 0.010 \\
\hline
\end{tabular}

The uncertainty expected in the measurement of the thermal conductivity is around 20\% [5]. The difference between the measurement in vacuum and in helium is $0.168 \pm 0.04 \mathrm{Wm}^{-1} \mathrm{~K}^{-1}$, while the thermal conductivity of helium gas at room temperature is $0.151 \mathrm{Wm}^{-1} \mathrm{~K}^{-1}$ The difference between the measurements in vacuum and air is $0.013 \pm 0.014 \mathrm{Wm}^{-1} \mathrm{~K}^{-1}$, while the air thermal conductivity at room temperature is $0.026 \mathrm{Wm}^{-1} \mathrm{~K}^{-1}$. Both results obtained are in agreement within their uncertainties.

\section{Theory}

From [6], the apparent thermal conductivity of graphite felt, $k_{f}$, is given by

$$
k_{f}=\frac{\left(k_{g c}+k_{f c}+k_{g r}+k_{f r}\right)}{1-f}+k_{s c}
$$

where $k_{s c}, k_{g c}, k_{f c}, k_{g r}$ and $k_{f r}$ are the thermal conductivities due to solid conduction along the fibre paths, gas conduction, free convection, gas radiation, and radiation exchanges between the fibres respectively.

In our application, the graphite felt is purged with nitrogen, argon or helium in order to avoid any ignition. These gases are transparent to infrared radiation and do not emit significantly [7, p464]. Therefore heat transfer by gas radiation is negligible and consequently we take $k_{g r} \approx 0$.

The Rayleigh number for free convection in a small cavity has been calculated to be less than 0.35 [8, p562], and therefore we take $k_{f c} \approx 0$

\section{Gas Conduction}

The three inert gases used to purge the graphite felt during operation are helium, argon and nitrogen. The thermodynamic properties of these gases can be easily found in the literature.

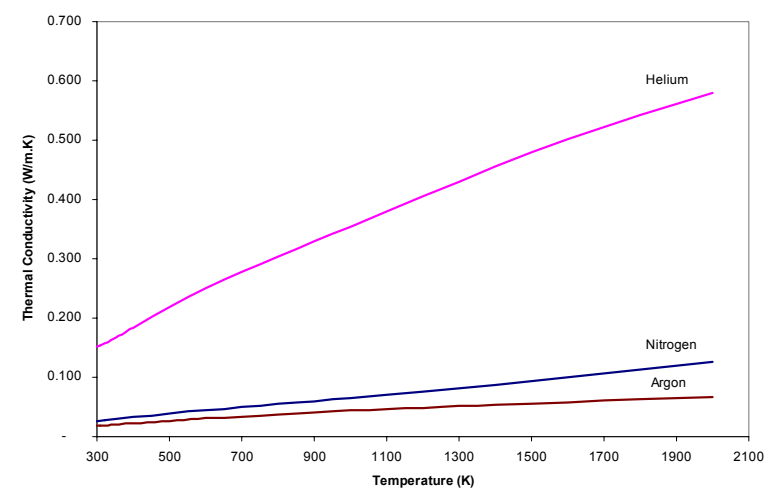

Fig. 3. Thermal conductivities of helium, nitrogen and argon versus temperature.

The thermal conductivities [9] of these gases at temperatures up to $2000 \mathrm{~K}$ are plotted in Figure 3, and were modelled using fifth degree polynomial functions of the absolute temperature $T$ with the following form

$$
k_{g c}(T)=c_{5} T^{5}+c_{4} T^{4}+c_{3} T^{3}+c_{2} T^{2}+c_{1} T+c_{0}
$$

where $c_{0}$ to $c_{5}$ are constant coefficients. The values we obtained for these coefficients for helium, argon and nitrogen are given in Table 2.

Table 2. Polynomial coefficients for helium, argon and nitrogen.

Table 2. Polynomial coefficients for helium, argon and nitrogen.
\begin{tabular}{l|c|c|c} 
& Helium & Nitrogen & Argon \\
\hline$c_{5}$ & $-2.3363 \times 10^{-18}$ & $-5.0736 \times 10^{-19}$ & $-1.0250 \times 10^{-18}$ \\
\hline$c_{4}$ & $-2.9313 \times 10^{-14}$ & $-7.8019 \times 10^{-15}$ & $4.6553 \times 10^{-15}$ \\
\hline$c_{3}$ & $1.7470 \times 10^{-10}$ & $4.9661 \times 10^{-11}$ & $-2.9810 \times 10^{-12}$ \\
\hline$c_{2}$ & $-3.4439 \times 10^{-7}$ & $-8.9523 \times 10^{-8}$ & $-1.7708 \times 10^{-8}$ \\
\hline$c_{1}$ & $5.4519 \times 10^{-4}$ & $1.1486 \times 10^{-4}$ & $5.9051 \times 10^{-5}$ \\
\hline$c_{0}$ & $1.1565 \times 10^{-2}$ & $-1.8605 \times 10^{-3}$ & $1.5875 \times 10^{-3}$
\end{tabular}

The maximum residuals of helium, nitrogen and argon using these models are $1.6,0.5$ and $0.8 \%$ respectively.

\section{Conduction along the Solid Paths}

This heat transfer mechanism is caused by conduction along the solid paths of the fibres, where heat transfers from one fibre to another across the contact points. As concluded by Bardon et al [10], most of the models 
developed for this mechanism in fibrous materials are purely empirical, their geometrical representation of the material texture is vague.

Bardon et al gave two equations to determine the conduction thermal conductivity of felt. The first equation was for the thermal conductivity of perfectly stratified felt, $k_{P S F}$, and is given as

$$
k_{P S F}=\frac{1.5 k_{S}(T)(f / \tau)^{2}}{r_{c}-0.838+0.231\left(\tau^{2} / f\right)}
$$

where $k_{s}(T)$ is the fibre thermal conductivity as a function of the absolute temperature $T, r_{c}$ is the ratio of fibre radius to contact spot radius, and $\tau$ is the tortuosity (ratio of the actual length of the fibre to the distance between the two contact points of this fibre). The second equation is for the imperfectly stratified felt (ISP); it took into consideration the case of fibres crossing from one layer to another with a frequency $x$ estimated by Bardon et al between 0.13 and 0.15 . The equation is given by

$$
k_{I S F}=(1-x) k_{P S F}(T)+\frac{4 f^{2} k_{s}(T) x}{\pi \tau^{3}}
$$

At room temperature, values for $k_{s}(T)$ can vary from 50 to $1400 \mathrm{Wm}^{-1} \mathrm{~K}^{-1}$ [11]. The thermal conductivity of our felt fibres is not known, but it exhibits the same patterns as graphite materials such as ATJ or ATJS. Figure 4 is a plot of ATJS graphite thermal conductivity versus temperature. Also, the thermal conductivity is given as a function of the absolute temperature $T$.

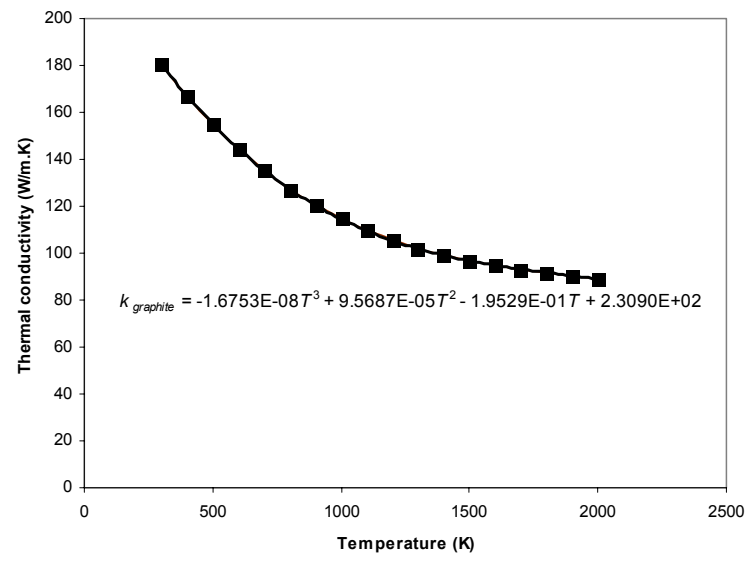

Fig. 4. Thermal conductivity of graphite versus temperature with the best fit curve function.

The value for $r_{c}$ varies from 500 to 2000 [10]. These values were obtained by estimating the contact spot radius as being between 5 and $20 \mathrm{~nm}$ and the measured radius of the fibre, $10.5 \mu \mathrm{m}$. This variation will lead to less than $1 \%$ variation in the conduction thermal conductivity.

Figure 5 is a plot of the conduction thermal conductivity versus temperature for different values of $\tau$ and $x$. The maximum change in the conduction thermal conductivity due to the maximum change in $x$ does not exceed $10 \%$. On the other hand, the conduction thermal conductivity is very sensitive to variation in the tortuosity; a $10 \%$ change in $\tau$ leads to an almost $25 \%$ drop in thermal conductivity.
According to Bardon et al, the value of $\tau$ cannot be measured directly due to the geometrical complexity of the felt structure. In order to determine the tortuosity value, the thermal conductivity of our graphite felt needed to be directly measured in vacuum.

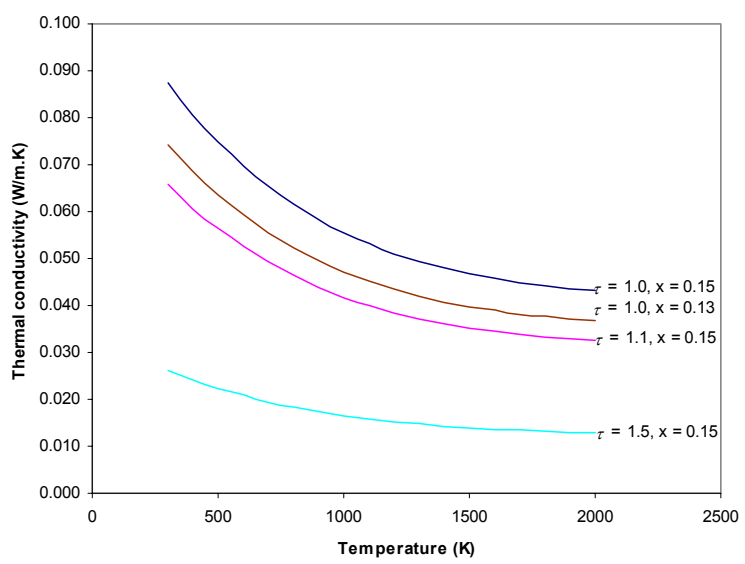

Fig. 5. Conduction thermal conductivity versus temperature for different values of $x$ and $\tau$.

In the absence of any gas at room temperature, heat transfer occurs by conduction only. Using equations (4.a) and (4.b), the measurement in vacuum given in Table 1 , and assuming a value of $x=0.15$ and $r_{c}=500$, the tortuosity ratio $\tau$ was calculated to be 1.11. Substituting the values of $\tau$ into equation (4.b) leads to the following equation

$$
k_{I S F}(T)=3.5 \times 10^{-4} k_{s}(T)
$$

which is the thermal conductivity due to conduction along the solid paths for our graphite felt, $k_{s c}(T)$.

\section{Radiative Exchanges}

In felt the dominating mechanism for heat transfer at high temperature is radiative exchanges. According to Tong et al [12], the radiative thermal conductivity is proportional to $\frac{r}{f} T^{3}$, and therefore the radiative thermal conductivity can be given as

$$
k_{f r}=C_{f r} \sigma \frac{r}{f} T^{3}
$$

where $C_{f r}$ is a radiation constant to be determined experimentally, and $\sigma$ is the Stefan-Boltzmann constant. Experimental results are needed in order to determine the radiation constant $C_{f r}$. Measurements were carried out by Lutkov et al [13] on graphite fibres and the results are plotted in Figure 6. The thermal conductivities at room temperature found by Lutkov et al in vacuum and in helium are 0.057 and $0.220 \mathrm{Wm}^{-1} \mathrm{~K}^{-1}$ respectively. From Table 1, our results for the vacuum and helium values are 0.064 and $0.232 \mathrm{Wm}^{-1} \mathrm{~K}^{-1}$ respectively. Hence, the felt used by Lutkov et al is similar to WDF felt, and therefore Lutkov et al's data can be used to calculate $C_{f r}$.

From Lutkov et al, the thermal conductivity in vacuum at a temperature of $900 \mathrm{~K}$ is $0.166 \mathrm{Wm}^{-1} \mathrm{~K}^{-1} . C_{f r}$ was consequently calculated using equations (2), (5) and (6) to be 15.0 . 


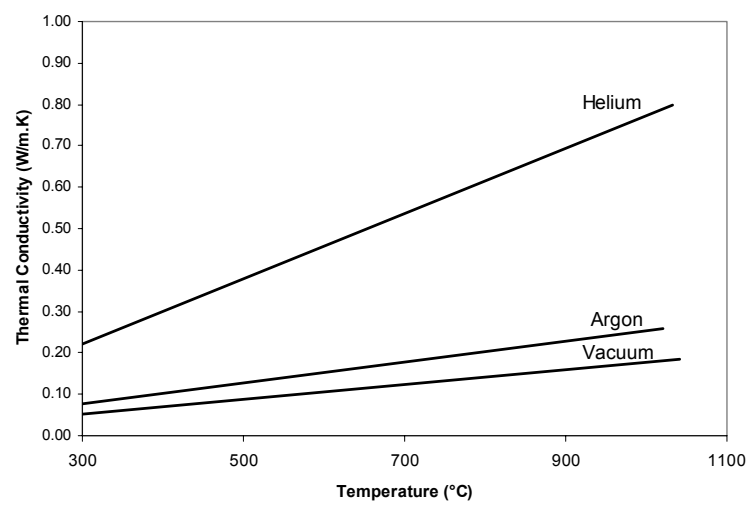

Fig. 6. Thermal conductivity of graphite felt given by Lutkov et al.

Final Equation for the Overall Thermal Conductivity

The apparent thermal conductivity of our graphite felt given in equation (2) can now be rewritten to include equations (5) and (6) with the calculated value of $C_{f r}=15.0$ to be

$$
k_{f}(T)=\frac{k_{g c}(T)+15.0 \sigma \frac{r}{f} T^{3}}{1-f}+3.5 \times 10^{-4} k_{s}(T)
$$

This equation can be used to calculate the apparent thermal conductivity at any temperature $T$. In addition, equation (7) can also be used to calculate the apparent thermal conductivity for felt with a different radius and volume fraction. Figure 7 shows the apparent thermal conductivity versus the absolute temperature for two felts with densities of 50 and $80 \mathrm{~kg} / \mathrm{m}^{3}$ using equation (7). The heat transfer mechanisms for both felts are also plotted.

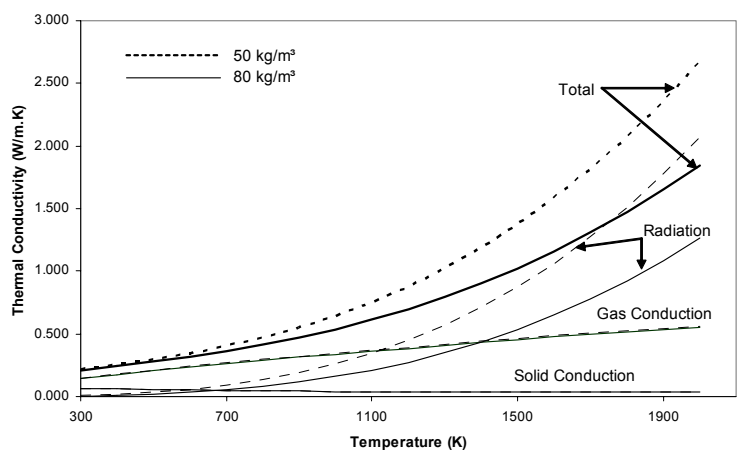

Fig. 7. Plot of calculated apparent thermal conductivities and their heat transfer mechanisms versus temperature for two graphite felts of different densities.

At low temperatures (less than $500 \mathrm{~K}$ ), the felt thermal conductivity is dominated by the solid and gas conduction mechanisms. At higher temperature, radiation and to some extent gas conduction (based on the gas used) dominate heat transfer in the felt. The radiative heat transfer mechanism is very sensitive to any change in the felt density, but other heat transfer mechanisms do not change substantially.

\section{Conclusions}

The determination of thermal conductivity of WDF graphite felt is complicated and requires a combination of theory and experiments. While the gas conduction heat transfer mechanism is fairly straightforward and values for the gases thermal conductivities could be obtained easily, the conduction and radiative mechanisms proved to be more complex and problematic due to the complex geometry of the felt. Most of the conductivity models are still purely empirical and they could not predict the conduction thermal conductivity without resorting to experimental results. Similarly, all of the radiative mechanism models reviewed have geometrical factors to be determined experimentally. As a conclusion, the apparent thermal conductivity of the WDF felt cannot be obtained on a purely theoretical basis. We have developed an empirical model for the thermal conductivity of graphite felt allowing for different densities, fibre diameters and gas conduction as a function of temperature.

\section{References}

1. Ballico, M. J. (1996). "Modelling of the effective emissivity of a graphite tube black body." Metrologia 32: 259-265.

2. ThermoGauge (2003). http://www.thermogauge.com/ ThermoGauge Instruments, Inc-Precision Blackbodies.

3. National Specialty Products (2004). http://www.nationalspecialties.com/, Carbon and Graphite.

4. Mantell, C. L. (1968). Carbon and graphite handbook. New York, Interscience Publishers.

5. Schneider, A. (1998). Thermal Conduction. Temperature and Humidity Measurement. R. E. Bentley. Singapore, Springer-Verlag. 1: 163-183.

6. Verschoor, J. D., P. Greebler, et al. (1951). "Heat transfer by gas conduction and radiation in fibrous insulations." The American Society of Mechanical Engineers, Atlantic City, USA, ASME.

7. Siegel, R. and J. R. Howell (2002). Thermal radiation heat transfer. London, Taylor \& Francis.

8. Incropera, F. P. and D. P. DeWitt (2002). Fundamentals of heat and mass transfer. New York, Wiley.

9. Vargaftik, N. B. (1983). Handbook of physical properties of liquids and gases : pure substances and mixtures. Washington, Hemisphere Pub. Corp.

10. Bardon, J.-P. and F. Danes (1994). "Heat transfer by solid conduction in a felt made of carbon fibre." High Temperatures - High Pressures 26: 323-330.

11. Taylor, R., S. P. Turner, et al. (1993). "Thermal conductivity of carbon fibres." High Temperatures High Pressures 25: 443 - 450.

12. Tong, T. W. and C. L. Tien (1980). "Analytical models for thermal radiation in fibrous insulations." Journal of Thermal Insulation 4: 27.

13. Lutkov, A. I., G. N. Zav'yalova, et al. (1975). "Thermal Conductivity of Carbon Felt and Lamp Black." High Temp 13(6): 1104-1107. 



\section{PREFACE}

The Australasian Heat and Mass Transfer Conference is tri-annual research forum in Australia organised under the auspices of the Australasian Fluid and Thermal Engineering Society (AFTES) for disseminating new knowledge to the global research community. This conference series covers both fundamental and applied topics in broad areas of convection, conduction, radiation, turbulence, multi-phase flow, combustion, drying, heat exchangers, computational methods, experimental methods, and other significant thermal processes in environmental, industrial, fire studies. The Eighth Australasian Heat and Mass Transfer Conference (8AHMTC) was held during 26-29 July 2005 at Curtin University of Technology in Perth, Western Australia.

As a research forum, 8AHMTC demonstrated a great success particularly in view of the tight timeframe and many logistical hurdles confronted by the organising committee. The conference attracted international participants from a range of countries including USA, Japan, Italy, Aus tralia and Singapore. 5 keynote lectures were delivered and 44 contributed papers were presented at the conference. As a testament to the quality, nine of 8AHMTC papers have been chosen and published in the Australian Journal of Mechanical Engineering (AJME), Vol. 4, No. 1.

8AHMTC was a very timely event for the State of Western Australia whose engineering industry enjoys a "resource boom" with unprecedented demand within the State for heat and mass transfer expertise. The conference theme readily captured this rejuvenated attention from engineering profession with wider participation and acted as a catalyst for future industry-academic partnerships. 8AHMTC also made a distinctive mark as the first-ever heat and mass transfer held in Western Australia.

The success of 8AHMTC culminated through the efforts of many involved. We wish to acknowledge the unyielding commitment from the local organising members that transpired into a conference of exemplary quality. Also, we express our deepest gratitude for the international advisory committee for immensely valuable advice and the panel of reviewers who undertook the onerous task of assessing the submitted papers for acceptance. We profusely thank Prof John Reizes of University of Technology Sydney and Prof Tony Lucey of Curtin University of Technology for their advice and guidance, and Mr Arun d'Souza of Engineers Australia for facilitating industry involvement. We also gratefully acknowledge the enthusiastic participation by the keynote speakers, Prof Richard Goldstein (USA), Prof Gian Piero Celata (Italy), Prof Nihal Wijeysundera (Singapore), Prof Ryosuke Suzuki (Japan) and Prof Steven Armfield (Australia), who added distinction to the conference, and all the other authors who inspirationally presented the ir work.

Finally, we wish to thank the conference sponsors, Australasian Fluid and Thermal Engineering Society (AFTES), Curtin University of Technology and Engineers Australia for providing financial and in-kind support, and Begell House Inc. for proceedings publication.

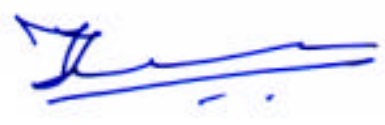

Tilak T Chandratilleke

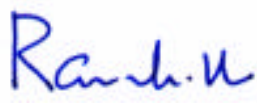

Ramesh Narayanaswamy

Editors- $8^{\text {th }}$ Australasian Heat and Mass Transfer Conference

26-29 July 2005, Curtin University of Technology, Perth, Western Australia 